\title{
De la mesure sur le dosimètre à la dose reçue par le porteur
}

\author{
A. BIAU
}

Comme indiqué au chapitre 1 , on se base dans la pratique courante sur un dosimètre porté au niveau de la poitrine pour évaluer la dose efficace et un dosimètre de complément pour déterminer la dose équivalente à un organe plus particulièrement exposé (mains, thyroïde, abdomen). Il est en effet impossible de faire porter un grand nombre de dosimètres comme nous l'avons montré au chapitre 1 . Une idée un peu baroque consistait à faire porter un habit dosimétrique constitué de tissu dosimétrique de sorte qu'on on aurait pu mesurer les doses reçues à l'ensemble du corps, cette idée lumineuse n'a pas eu de prolongements !

Malgré tout, en dépit des incertitudes liées à la mesure elle-même et à la représentativité de la mesure de dose sur le dosimètre par rapport à la dose réellement reçue par le porteur, la surveillance dosimétrique individuelle mise en place il y a un demi-siècle a permis de limiter l'exposition des travailleurs de façon de plus en plus efficace, bien mieux que dans d'autres types de nuisances physico-chimiques.

\subsection{Les incertitudes liées à la mesure}

Il y a tout d'abord l'incertitude sur la mesure physique, densité optique sur les films, quantité de lumière sur les dosimètres luminescents, qui sont de l'ordre du pourcent.

L'autre incertitude dépend de la réponse en énergie du dosimètre et de la qualité des étalonnages. Ce type d'incertitude est limité lorsque les conditions d'exposition sont connues grâce à des études de poste dans des installations comme au CEA, à AREVA ou au CNRS, dans ce cas l'incertitude peut se limiter à moins de $10 \%$ pour les rayonnements $\gamma$ et $\mathrm{X}$.

Par contre pour les laboratoires généralistes comme l'IRSN ou LCIE, lorsque les conditions d'exposition sont moins connues et variables d'un établissement à l'autre, l'incertitude est plus importante surtout lorsque l'énergie des rayonnements est très élevée ou très faible par rapport aux énergies auxquelles les dosimètres sont soumis lors des étalonnages. La présence d'écrans dans le support des dosimètres 
permet d'apprécier la nature et l'énergie du rayonnement mais avec une incertitude qui peut atteindre $50 \%$ pour les rayonnements $\gamma$ et $X$ de faible énergie.

\subsection{Les incertitudes liées à l'assimilation de la dose du dosimètre à la dose du porteur}

La situation théorique où la dose mesurée sur le dosimètre est égale à la dose efficace reçue par le porteur n'est jamais conforme à la réalité.

Les raisons de ces incertitudes sont :

- l'inhomogénéité du champ de rayonnements ;

- la position de la source de rayonnements par rapport à l'individu et son dosimètre (voir figure 3.1) ;

- la façon dont le dosimètre est porté, dans la poche poitrine ou à l'extérieur du vêtement.

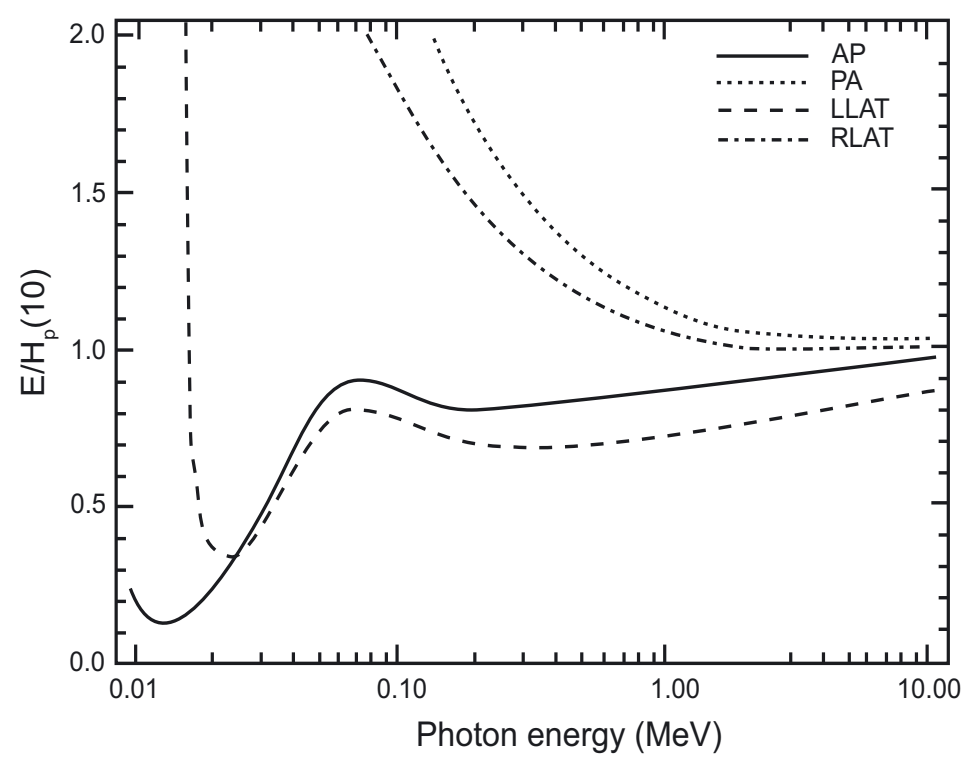

Figure 3.1 - Variation du rapport entre la dose efficace et l'équivalent de dose mesuré sur le dosimètre en fonction de l'énergie des photons et l'angle d'incidence (Health Physics, 1999).

Sur ces graphiques, on considère que le dosimètre est porté au niveau de la poitrine côté gauche et l'on évalue les rapports entre la mesure du dosimètre et la dose efficace en fonction de l'énergie d'un rayonnement $\gamma$ monochromatique 
d'énergie comprise entre $10 \mathrm{keV}$ et $10 \mathrm{MeV}$. Ces courbes ont été réalisées pour les incidences antéropostérieure (AP), postéro-antérieure (PA), latérale gauche (LLAT) et latérale droite (RLAT).

Aux énergies inférieures à $100 \mathrm{keV}$, les écarts sont importants entre les quatre incidences, plus modérés entre $100 \mathrm{keV}$ et $1 \mathrm{MeV}$ et très corrects au dessus de $1 \mathrm{MeV}$.

Pour tenir compte de toutes ces incertitudes, la CIPR, dans sa publication $\mathrm{n}^{\circ} 35$ (ICRP, 1982), considère comme acceptables des résultats compris entre $D / 2$ et $2 D$ pour une dose $D$ inférieure à $1 \mathrm{mSv}$ et, des résultats compris entre $D / 1,5$ et $1,5 D$ pour une dose supérieure à $1 \mathrm{mSv}$.

\section{RÉFÉRENCES}

Health Physics (1999) Health Physics Report February 1999, Volume 76, Number 2.

ICRP Publication 35 (1982) General Principles of Monitoring for Radiation Protection of workers, Ann. $I C R P, \mathrm{n}^{\circ} 4$. 